\title{
Conocimiento y práctica de autocuidado del profesional de Enfermería del servicio de Pediatría del Hospital Nacional Guillermo Almenara Irigoyen, 2013
}

\author{
Knowledge and practice of self-care nurse service Pediatrics, Hospital Nacional Guillermo \\ Almenara Irigoyen, 2013
}

Zenaida Doylitt Manturano Velásquez¹, María Teresa Cabanillas Chávez²

\begin{abstract}
RESUMEN
Objetivo: Determinar la relación entre conocimiento y práctica de autocuidado del profesional de Enfermería en el servicio de Pediatría del Hospital Nacional Guillermo Almenara Irigoyen. Material y método: La investigación fue de tipo descriptivo correlacional, cuantitativa, de diseño no experimental, de corte transversal; la muestra estuvo constituida por 45 enfermeras. Para la recolección de datos se utilizó dos cuestionarios diferentes adaptados por la investigadora, para el área de la práctica se utilizó preguntas de "Escala de Valoración de la Capacidad de Agencia de Autocuidado" desarrollado por Ysenberg y Evers, traducida y validada al español por Esther Gallegos; y para la variable conocimiento la investigadora elaboró un cuestionario que fue validado por expertos en el área. Resultados: Los resultados alcanzados fueron que un $42.2 \%$ de las enfermeras no conocen sobre autocuidado y muestra una práctica inadecuada sobre el mismo; de igual forma, un $26.7 \%$ está en la escala de conocer y practica adecuadamente su autocuidado; asimismo, existe un $8.9 \%$ del profesional de Enfermería que, a pesar de no conocer sobre el autocuidado, tiene prácticas adecuadas de autocuidado. Aplicando la prueba estadística de chi cuadrado, la relación de las variables fue significativa $\mathrm{p}=0.009$, indicando que existe relación significativa entre ambas variables. Conclusión: Existe relación significativa entre el conocimiento y la práctica que el profesional de Enfermería posee sobre su autocuidado.
\end{abstract}

Palabras clave: Conocimiento, práctica, autocuidado y profesional de Enfermería.

\begin{abstract}
Objective: To determine the relationship between knowledge and practice self-care nurse in the pediatric ward of the National Hospital Guillermo Almenara Irigoyen. Methods: The research is correlational, descriptive quantitative, non experimental design, cross-section; the sample consisted of 45 nurses. Two different questionnaires adapted by the researcher, for the area of practical questions "Rating Scale Capacity Agency Self-Care" developed by Ysenberg and Evers, translated and validated in Spanish by Esther was used was used for data collection Gallegos; and to the knowledge variable researcher developed a questionnaire, which was validated by experts in the field. Results: The results obtained were that $42.2 \%$ of the sample is within the range of not knowing about self and displays an appropriate practice on it; likewise $26.7 \%$ is in the range of proper understanding and practice self-care; there is also a $8.9 \%$ of the nurse who despite not knowing about the self has adequate self-care practices. Applying statistical chi square test the relationship of the variables was significant $\mathrm{p}$ $=0.009$, indicating that there is significant relationship between both variables. Conclusion: There is significant relationship between knowledge and practice that the nurse has on yourself.
\end{abstract}

Keywords: knowledge, practice, self-care and professional nursing. 


\section{INTRODUCCIÓN}

El autocuidado, en la actualidad, constituye un tema de trascendencia, porque mediante su conocimiento y aplicación permiten incrementar la proactividad de los trabajadores en el campo de la salud. La práctica del autocuidado requiere, en gran medida, cambio de actitud frente a los nuevos retos de la vida moderna (Uribe, 2003).

Las nuevas concepciones de salud y promoción de la salud proponen una nueva forma de asumir el autocuidado con rostro humano, como estrategia fundamental para promover la vida y el bienestar; por eso se requiere el desarrollo de habilidades personales para optar decisiones saludables, lo cual constituye uno de los cinco componentes de la promoción de la salud (Brunner y Suddarth, 1998).

Dentro del área de la salud surgen diversos enfoques de autocuidado, se destaca el de la enfermera Dorotea Orem (1999), quien propone la teoría de autocuidado, interrelaciona tres teorías: el autocuidado, el déficit de autocuidado y los sistemas de enfermería. Esta teoría expresa la práctica de actividades que los individuos realizan por sí y para sí mismos, para mantener la vida, la salud y el bienestar (De la Fuente, 2011).

El profesional de enfermería es el responsable del fomento del autocuidado en las diferentes etapas de vida de las personas, tanto con su testimonio de vida sana como con la educación para la salud, es una herramienta fundamental para los cambios en las conductas de las personas (Tobón, 2004). Con la intervención educativa se facilita la integración y participación del paciente y familia, fomentando en todo momento la toma de decisiones. De tal manera, la enfermera es la facilitadora de información y de las estrategias para lograr el autocuidado.

El profesional de enfermería, gracias a su formación recibida, conoce sobre el autocuidado y los estilos de vida saludables, sin embargo, estos conocimientos no garantizan que este grupo ocupacional aplique el autocuidado a través de sus propios estilos de vida saludable, debido a los factores individuales y del entorno a los que están expuestas, especialmente a sus propios hábitos, costumbres y actitudes, para lo cual es necesario internalizar la importancia de la responsabilidad y el compromiso que se tiene consigo mismo (Uribe, 2003).

Existe un desmedro en la salud del usuario interno a nivel organizacional; es decir, las políticas de salud no promueven estilos de vida saludables para los trabajadores; por ejemplo, ambiente físico, clima laboral y condiciones de trabajo.

La desvalorización del autocuidado tiene un componente individual; es decir, aunque se tenga mejores condiciones para trabajar, la promoción de la salud es escasa. Es así como se observa las paradojas comportamentales, escritas por De Roux G. y estas se definen como comportamientos nocivos que tienen las personas con conocimientos saludables (Diplomado en Salud Pública Módulo I: Tendencias en Salud Pública: Salud y Salud Familiar y Comunitaria y Promoción).

El presente estudio ofrece un enfoque clínico y social para realizar un diagnóstico situacional sobre los conocimientos que poseen las enfermeras, cuyos "estilos de vida saludables" de una persona son elementos fundamentales, tienen gran influencia en su desempeño y realización, les permite tener un autocuidado para conservar su salud y disminuir el riesgo de padecer enfermedades, así mismo serán más eficientes en el quehacer de enfermería (Carrillo y Amaya, 2001).

En tal sentido, en el Perú, el Ministerio de Salud y otras organizaciones no gubernamentales incentivan la promoción de la salud que va más allá de la transmisión de contenidos e incorpora los objetivos de fortalecer las habilidades y capacidades de los individuos y de modificar las condiciones sociales, ambientales y económicas, con el propósito de disminuir su impacto negativo en la salud individual y de la comunidad. Por lo que a nivel nacional se busca continuar investigaciones al personal de salud para contribuir a mejorar la salud de la población.

Las enfermeras del servicio de Pediatría del HNGAI no se encuentran exentas de esta realidad, pues los estilos de vida de este grupo deberían ser óptimos; sin embargo, frecuentemente existen descansos médicos, debido a diferentes problemas de salud, perjudicándose a sí mismas y a su entorno. Por tal motivo, la investigadora se propuso determinar la relación entre conocimiento y práctica de autocuidado del profesional de enfermería del servicio de Pediatría del Hospital Nacional Guillermo Almenara Irigoyen.

En este estudio se realizó una revisión bibliográfica sobre los temas relacionados con autocuidado en las diferentes dimensiones y se enfatizó el tema de las enfermedades no transmisibles, porque en la actualidad las estadísticas muestran altas tasas de morbi-mortalidad por estas enfermedades relacionadas con los estilos de vida; es decir, a la falta de autocuidado en el ser humano. 
El estudio fue de tipo cuantitativo, correlacional, de diseño no experimental, de corte transversal. Se ha elaborado dos cuestionarios para la recolección de datos referidos al conocimiento y la práctica del autocuidado respectivamente, cada uno con las dimensiones: biológica, psicológica, social y espiritual; aplicándolos a una muestra de 45 enfermeras del servicio de Pediatría del Hospital Nacional Guillermo Almenara Irigoyen.

La presente investigación es un aporte para mejorar la calidad de vida del profesional de enfermería del servicio de Pediatría. Asimismo, realzar el nivel de la profesión mediante el fortalecimiento de la investigación en enfermería; los resultados obtenidos sirven de guía para establecer propuestas de programas preventivos para la población de profesionales en salud, y así disminuir los factores de riesgo en su salud.

\section{MATERIAL Y MÉTODOS}

El presente estudio de investigación es de tipo descriptivo, correlacional, cuantitativo, de diseño no experimental, de corte transversal, la población de estudio estuvo constituida por todas las enfermeras del servicio de Pediatría del HNGAI, siendo un total de 45 enfermeras, considerándose una muestra no probabilística al $100 \%$ de la población, por conveniencia de la autora debido a que la población es pequeña. Como instrumento se utilizó dos cuestionarios, los cuales se aplicaron a la muestra de estudio en un mismo momento. En la elaboración del instrumento, para el área de práctica, se utilizó algunas preguntas de la "Escala de Valoración de la Capacidad de Agencia de Autocuidado" desarrollado por Ysenberg y Evers en 1993, traducida y validada en español por Esther Gallegos en 1998, esta escala se derivó del concepto de Capacidades de Autocuidado desarrollado por Dorothea Orem. La validación del cuestionario "Práctica del autocuidado" se realizó a través de la escala Alfa de Cronbach, dando un estadístico de fiabilidad de 0,759. Para el cuestionario sobre "conocimiento de autocuidado" se realizó a través del juicio de expertos, quienes fueron elegidos por el investigador, de acuerdo con la experiencia del profesional sobre el tema de investigación.

\section{RESULTADOS}

Tabla 1

Datos sociodemográficos del profesional de enfermería del servicio de Pediatría del Hospital Nacional Guillermo Almenara Irigoyen, 2013.

\begin{tabular}{llll}
\hline Datos sociodemográficos: & N. & $\%$ \\
\hline \multirow{2}{*}{ Procedencia } & Costa & 15 & 33.3 \\
& Sierra & 27 & 60.0 \\
& Selva & 3 & 6.7 \\
\multirow{4}{*}{ Edad } & 26 años - 36 años & 15 & 33.3 \\
\multirow{5}{*}{ Nivel académico } & 37 años - 47 años & 18 & 40.0 \\
& 47 años - 57 años & 12 & 26.7 \\
& Licenciada & 9 & 20.0 \\
Años de servicio & Especialista & 25 & 55.6 \\
& Estudio de maestría & 11 & 24.4 \\
& 2 años - 10 años & 14 & 31.1 \\
& 11 años - 20 años & 20 & 44.4 \\
\hline
\end{tabular}

El presente estudio ha sido aplicado en una población $100 \%$ de sexo femenino. En la presente tabla evidenciamos que, de la población en estudio, el $60 \%$ procede de la sierra, el $33.3 \%$ procede de la costa y solo el $6.7 \%$ procede de la selva; en relación con la edad, el $40 \%$ oscila entre los 37 y 47 años, el $33.3 \%$ entre 26 y 36 años de edad y el $26.7 \%$ entre 47 a 57 años de edad; en relación con respecto al nivel académico, el $55.6 \%$ tiene especialidades en diferentes áreas de enfermería, el $24.4 \%$ tiene estudios de maestría y el $20 \%$ de la población en estudio continúan como enfermeras generales, ya que solo tienen el grado de licenciada y, finalmente, el $44.4 \%$ tiene de 11 a 20 años de servicio en la institución, asimismo, solo el $24.4 \%$ tiene de 21 a 29 años de servicio. 
Tabla 2

Conocimiento de Autocuidado en las dimensiones: biológica, psicológica, social y espiritual del profesional de enfermería del servicio de Pediatría del Hospital Almenara Irigoyen, 2013.

\begin{tabular}{lllll}
\hline \multirow{2}{*}{ Conocimiento de autocuidado en las dimensiones: } & \multicolumn{2}{c}{ Conoce } & \multicolumn{2}{c}{ No conoce } \\
\cline { 2 - 5 } & N. ${ }^{\circ}$ & $\%$ & N. & $\%$ \\
\hline Biológica & 18 & $40.0 \%$ & 27 & $60.0 \%$ \\
Psicológica & 11 & $24.4 \%$ & 34 & $75.6 \%$ \\
Social & 11 & $24.4 \%$ & 34 & $75.6 \%$ \\
Espiritual & 20 & $44.4 \%$ & 25 & $55.6 \%$ \\
\hline
\end{tabular}

En la tabla 2 evidenciamos que, en relación con la dimensión psicológica, se presenta un $75.6 \%$ de enfermeras que no conoce sobre autocuidado, con el mismo porcentaje se muestra, en la dimensión social, el desconocimiento del personal encuestado sobre el autocuidado; también podemos observar que solo el
$40 \%$ del total de enfermeras del servicio de pediatría conoce de autocuidado en la dimensión biológica, y el $60 \%$ de del personal desconoce del autocuidado en la dimensión mencionada. Asimismo, evidenciamos que existe un $44.4 \%$ que tiene conocimiento sobre autocuidado en la dimensión espiritual.

Tabla 3

Práctica de Autocuidado en las dimensiones: biológica, psicológica, social y espiritual del profesional de Enfermería del servicio de Pediatría, Hospital Almenara Irigoyen, 2013.

\begin{tabular}{lllll}
\hline \multirow{2}{*}{ Práctica de autocuidado en las dimensiones } & \multicolumn{3}{c}{ Adecuada } & \multicolumn{2}{c}{ Inadecuada } \\
\cline { 2 - 5 } & $\mathrm{N} .{ }^{\circ}$ & $\%$ & $\mathrm{~N} .{ }^{\circ}$ & $\%$ \\
\hline Biológica & 15 & $33.3 \%$ & 30 & $66.7 \%$ \\
Psicológica & 12 & $26.7 \%$ & 33 & $73.3 \%$ \\
Social & 14 & $31.1 \%$ & 31 & $68.9 \%$ \\
Espiritual & 17 & $37.8 \%$ & 28 & $62.2 \%$ \\
\hline
\end{tabular}

En la presente tabla observamos que el $73.3 \%$ de profesional de enfermería encuestado tiene prácticas inadecuadas de autocuidado en la dimensión psicológica; asimismo, se presenta un $33.3 \%$ de enfermeras tiene prácticas adecuadas, en relación al autocuidado en la dimensión biológica; sin embargo, el $66.7 \%$ incurre en prácticas inadecuadas respecto a su autocuidado en esta dimensión. Del mismo modo, el $37.8 \%$ presenta prácticas adecuadas de autocuidado en la dimensión espiritual y el $62.2 \%$ tiene prácticas inadecuadas en su autocuidado. En relación con la dimensión social, solo el $31.1 \%$ de enfermeras encuestadas tiene prácticas adecuadas y la diferencia, o sea el $68.9 \%$, presenta prácticas inadecuadas en su autocuidado.

Tabla 4

Relación de conocimiento y práctica de Autocuidado del profesional de Enfermería en el servicio de Pediatría del Hospital Nacional Guillermo Almenara Irigoyen, 2013.

\begin{tabular}{llllll}
\hline \multirow{2}{*}{ Autocuidado } & \multicolumn{4}{c}{ Práctica } \\
\cline { 3 - 6 } & & Inadecuadas & \multicolumn{3}{c}{ Adecuadas } \\
\cline { 3 - 6 } & N. ${ }^{\circ}$ & $\%$ & N. ${ }^{\circ}$ & $\%$ \\
\hline \multirow{2}{*}{ Conocimiento } & No conoce & 19 & $42.2 \%$ & 4 & $8.9 \%$ \\
& Conoce & 10 & $22.2 \%$ & 12 & $26.7 \%$ \\
\hline
\end{tabular}

Nota: $\mathrm{X}^{2}=6.774^{\mathrm{a}} ; \mathrm{Gl}=1 ; \mathrm{sig}=0.009$

En la tabla 4 se observa que las prácticas adecuadas se relacionan con el conocimiento del autocuidado en un $26.7 \%$, mientras que las prácticas inadecuadas se relacionan con el desconocimiento del autocuidado del profesional de enfermería en $42.2 \%$. Asimismo, cabe resaltar que, a pesar de conocer las medidas de autocuidado, existe un alto porcentaje de prácticas inadecuadas $22.2 \%$; siendo que se espera 
que el porcentaje de prácticas inadecuadas fuese mucho menor. Por otro lado, se observa que, a pesar del desconocimiento de las medidas de autocuidado, existe un porcentaje considerable, $8.9 \%$, de prácticas adecuadas. El valor de chi cuadrado es significativo a nivel de 0.05; es decir, se acepta la hipótesis de investigación (alterna) con 0.009 ; por lo tanto, afirmamos que existe relación significativa entre el conocimiento y la práctica de autocuidado de los profesionales de enfermería del servicio de Pediatría del HNGAI.

\section{DISCUSIÓN}

El profesional de enfermería debe internalizar que la salud es consecuencia de un proceso dinámico y complejo; es decir, estar bien en todas las dimensiones del ser humano. En la medida de que el personal de salud viva con bienestar, está en condiciones de promocionar la salud de los demás "la palabra enseña y el ejemplo arrastra" (Uribe, 2003).

La enfermera es un profesional que ha adquirido competencia científico-técnica, para cuidar y ayudar a las personas sanas o enfermas; ya que el "conocimiento te da la capacidad de resolver un determinado conjunto de problemas con una efectividad determinada" (Abarca, 2012; citado por Tobón, 2012). Por tal motivo, es necesario ejercer el rol de profesional de la enfermería, como una persona productiva y eficiente, con responsabilidad social y comprometida con su entorno, porque actualmente en nuestra sociedad se observa el incremento de las enfermedades no transmisibles, originadas solo por falta de autocuidado, incluidos en esto el mismo personal de salud quienes caen fácilmente en esta situación; por ello, se debe concientizar para mejorar y actualizar los conocimientos, de ese modo actuar con mayor fuerza en la prevención y promoción de la salud.

En el presente estudio, en relación con el conocimiento de autocuidado en las dimensiones: biológica, psicológica, social y espiritual del profesional de enfermería del servicio de Pediatría, se observa que un alto porcentaje no conoce el autocuidado, mostrándose los porcentajes: $60 \%$, $75.6 \%, 75.6 \%$ y $55.6 \%$ respectivamente a las dimensiones mencionadas; a pesar de que el profesional de enfermería, como eje fundamental de la salud de la sociedad, debe estar capacitado en este tema, ya que la acción y efecto de conocer brinda entendimiento, inteligencia, razón natural que dará como resultado el proporcionar una atención de enfermería rentable de calidad que fomentará los estándares óptimos para el paciente y familia; y también se debe considerar que la principal preocupación de la enfermera(o), es y debe ser brindar una atención humanizada y de calidad para el paciente y familia, sin olvidarse de su propio cuidado (Romero, 2005).

En relación con la práctica de autocuidado en las dimensiones: biológica, psicológica, social y espiritual, se muestra que un $73.3 \%$ de las enfermeras encuestadas tiene prácticas inadecuadas, en relación con la dimensión psicológica, esto puede deberse a que se considera que, la formación profesional recibida por el personal de salud, puede ser un elemento que influya en forma decisiva en el tipo de autocuidado que este prodiga, ya que básicamente dan importancia a la salud física que a la salud mental y a otras áreas. Sin embargo, también se evidencia que un $33.3 \%$ de prácticas adecuadas se encuentra en la dimensión biológica, seguida de $37.8 \%$ que existe prácticas adecuadas en la dimensión espiritual, demostrándose así que el mayor porcentaje corresponde a prácticas inadecuadas; en ese sentido, también Hernández y otros en su estudio "Autocuidado en profesionistas de la salud y profesionistas universitarios" obtuvieron como resultado que los profesionistas de la salud incurren, en mayor porcentaje, en prácticas nocivas o al menos no anticipatorias para la salud, en comparación con otros profesionistas universitarios. Asumir una práctica de autocuidado se refiere a cuidarse a sí mismo, por lo que esta es una oportunidad de relación con uno y los otros de manera más cariñosa, reflexiva y consciente, donde la relación con el cuerpo es fundamental para reconocer patrones de conducta que interfieren en la salud mental, física, emocional y espiritual.

La conciencia corporal, mental, emocional y espiritual no solo previene el malestar, sino que promueve una mejor calidad de vida y bienestar humano (Arriagada y González, 2004).

Practicar el autocuidado es ejercer mejor control sobre su propia salud, expresar sus experiencias y dirigir sus esfuerzos, para participar y hacer cosas que son beneficiosas para su ser y para otros (Llado, 1998). Consiste también en organizar su tiempo y qué porción de este es destinado a sentirse mejor, en relación a cada dimensión de su ser.

Los resultados obtenidos permiten confirmar lo que Tobón (2004) afirmó sobre el conocimiento, que determina, en gran medida, la adopción de prácticas saludables, pues permiten a las personas tomar decisiones informadas y optar por prácticas saludables o de riesgo, frente al cuidado de su salud; sin embargo, estas determinaciones no dependen 
exclusivamente de la educación ni de la información, en vista de que hay variados factores internos y externos que intervienen; los factores internos pueden ser: la voluntad, las motivaciones, los hábitos y las actitudes; los factores externos pueden ser: la cultura, los estilos de vida, la tecnología, entre otros; todo ello se debe rescatar que cuando los factores internos y externos interactúan de manera favorable, se logra una buena calidad de vida (Orem, 1994).

Según este resultado, evidenciamos que existe relación entre conocimiento y práctica de autocuidado en el profesional de enfermería del servicio de Pediatría, mostrándose así que un $42.2 \%$ del personal desconoce sobre autocuidado y, por ende, tiene una práctica inadecuada; igualmente se observa que un $26.7 \%$ conoce del tema y tiene unas prácticas adecuadas de autocuidado. Hecho que se encuentra sustentado también en el estudio de investigación de Arrivillaga y Salazar (2005), "Creencias relacionadas con el estilo de vida de jóvenes latinoamericanos", una persona puede conocer o desconocer la importancia de cualquier comportamiento de protección o de riesgo para la salud. Si la desconoce, obviamente, las probabilidades de realización de una práctica saludable es menor que el desarrollo de una práctica poco o nada saludable.

En el presente estudio, según la prueba de chi cuadrado aplicado, se concluyó que es significativo a nivel de 0.05; es decir, se acepta la hipótesis de investigación (alterna) con 0.009; por lo tanto, afirmamos que existe relación significativa entre el conocimiento y la práctica de autocuidado de los profesionales de enfermería del servicio de Pediatría del HNGAI.

Uribe (2003) mencionó que, para el fomento del autocuidado el profesional de la salud, se requiere internalizar que la salud es consecuencia de un proceso dinámico, complejo; un proceso que depende del cuerpo, de la mente, de las emociones, de la vida espiritual y de las relaciones con los demás.

Por otra parte, la OMS (2001) mencionó que el aumento del conocimiento no asegura un cambio conductual, pero es importante, porque constituye el primer paso para el proceso del cambio. También Flynn, Slovic y Mertz (1994) corroboraron que los aspectos cognoscitivos son necesarios pero no suficientes, para que se desarrolle en el individuo una adecuada percepción, representan solo un eslabón en el proceso de percepción del riesgo y, por consiguiente, en el cambio de conducta. Todo esto es corroborado en el área de investigación, por lo que se evidencia un porcentaje alto de estudios sobre autocuidado dirigido hacia el padecimiento de una enfermedad; es decir, la mayoría de los seres humanos cambian o modifican su autocuidado en relación con el dolor o sufrimiento; por tal motivo, son pocos los estudios dirigidos a promover estilos de vida saludables en personas sin algún padecimiento.

La enfermería es una profesión que tiene dentro de su actuación el acogimiento del sufrimiento humano y a través del cuidado rehabilita, mitiga los dolores y promociona salud (Orem, 1994). Por eso se debe asumir que el autocuidado es una vivencia cotidiana y en la medida de que el personal de salud vive con bienestar, está en condiciones de promocionar la salud de los demás. "la palabra enseña y el ejemplo arrastra".

En el presente estudio se evidencia que un 20 $\%$ de enfermeras conoce sobre el autocuidado en la dimensión biológica; sin embargo, tiene una práctica inadecuada, lo que determina esta situación como un proceso que Wenstein (1988) denominó "Invulnerabilidad percibida"; la persona piensa y siente que no es vulnerable y no tiene ninguna posibilidad de desarrollar disfunción o enfermedad alguna; de igual forma, la persona puede estar adoptando un modelo conductual, donde ni siquiera piensa acerca de practicar una conducta saludable, lo cual trae consigo una actitud de indiferencia y hasta cierto punto negativo, sobre la práctica misma (Arrivillaga y Salazar, 2005).

Asimismo, también se considera que la población en estudio está constituida, en su totalidad, por personas del sexo femenino, hecho que tal vez permita entender por qué el conocimiento no es un determinante en el cambio de conducta o la aplicación de una práctica adecuada de autocuidado, ya que, según Colliere (1993), la mujer cumple el rol de cuidadora de otros, modelo femenino de ser para otros versus ser para sí; el cual ha generado en la mujer bajos niveles de autoconcepto, autonomía y autoestima, influyendo significativamente en la forma de asumir el autocuidado de su vida, su cuerpo y su salud. En este sentido, la cultura ha ido dejando huella mediante una serie de consecuencias nocivas para la asunción del autocuidado por parte de esta.

Si bien es cierto, el autocuidado en la dimensión biológica, en el ser humano, se basa en acciones que están asociadas generalmente a los cambios, deterioros o a una disminución en la eficiencia del funcionamiento orgánico y estas están relacionadas con las acciones dirigidas a los aspectos de la alimentación, ejercicio, sueño, respiración, circulación, eliminación y la higiene. También se 
debe pensar que existen varios cambios que están presentes en toda persona y que avanza a ritmos diferentes, estos dependen de los estilos de vida como es del consumo de alimentación saludable, plan de ejercicios, rutina de evacuación intestinal, entre otros (Quintero, 1997).

En la actualidad se ha ido evidenciando en la población que la práctica inadecuada en autocuidado, en esta dimensión, está incrementando las ENT; por lo cual, la OMS (2011) menciona que las enfermedades no transmisibles (ENT) constituyen las principales causas de muerte y discapacidad en el mundo y el rápido aumento de las ENT representa uno de los mayores retos para el desarrollo mundial en el presente siglo. Este creciente desafío amenaza el desarrollo económico y social, así como la vida y la salud de millones de personas.

\section{Declaración de financiamiento y de conflicto de} intereses:

El estudio fue financiado por la autora, quien declara no tener algún tipo de conflicto de interés en la investigación realizada.

\section{Correspondencia}

Zenaida Doylitt Manturano Velásquez Hospital Guillermo Almenara Irigoyen Av. Grau 800. Teléfono: 324-2983 / 324-2980 Correo electrónico: doyma23@hotmail.com 


\section{REFERENCIAS BIBLIOGRÁFICAS}

Alcántar, L. A. (2008). Factores que influyen en el autocuidado de enfermería en la preparación y manejo de citostáticos, Morelia, Michoacan - México (Tesis Maestría) Universidad Michoacana de San Nicolás de Hidalgo. México. Recuperado el 10 de agosto del 2013 de: http://bibliotecavirtual.dgb. umich.mx:8083/jspui/bitstream/123456789/3253/1/ FACTORESQUEINFLUYENENELAUTOCUIDADO DEENFERMERIAENLAPREPARACIONYMANEJO DECOTOSTATICOS.pdf

Arriagada \& González (2004). Documento de consejería y autocuidado, Universidad de Chile. Recuperado el 23 de enero 2013 de: box.jisko.net/d/6d292add

Arrivillaga, M. \& Salazar, I. (2005). "Creencias relacionadas con el estilo de vida de jóvenes latinoamericanos". Psicología conductual. 13(1), 19-36. Recuperado el 05 de mayo del 2013 de: http://javeriana.edu.co/redcups/ Investigacion_Creencias_y_\%20Estilos_de_vida.pdf

Brunner \& Suddarth. (1998). Manual de enfermería. 4. ${ }^{\mathrm{a}}$ Edición. Tomo II. México: Editorial Interamericana.

Carrillo, H. \& Amaya, M. (2001). Estilos de vida saludables de las enfermeras (O) docentes de la Escuela de Enfermería de la Universidad Pedagógica y Tecnológica de Colombia. (Tesis de Licenciatura). Universidad Pedagógica y Tecnológica de Colombia. Tunja. Recuperado el 23 de junio del 2013 de. http:// www.index-f.com/lascasas/documentos/lc0128.pdf

Colliere, MF. (1993). Promover la vida. Madrid: Interamericana.

De la Fuente, V., Omaña, V., Soon Ran, R., Alavés, C., Peña, H. \& Sierra, E. (2011). Conocimiento y acciones de los adolescentes sobre el autocuidado de su salud. Rev. CONAMED, 16(1) 29-33. Recuperado el 15 de mayo del 2013 de: http://www.conamed.gob. $\mathrm{mx} /$ publicaciones/pdf/REVISTA ENE-MAR 2011. pdf\#page $=31 \mathrm{http}: / /$ dialnet.unirioja.es/servlet/ articulo codigo $=3639980 \&$ orden $=293368 \&$ info $=$ link $\#$ page $=31$

Flynn, Slovic \& Mertz. (1994,) citado por Fernández, Varona, García, Ibarra y Chang (2000). Tabaquismo y sus características en trabajadores de la salud, Cuba. Recuperado el 23 de junio del 2013 de: http://scielo.sld. $\mathrm{cu} / \mathrm{scielo}$.php?script=sci_arttext\&pid=S0864-212520 000300001

Llado, A. (1998). La cuarta edad (después de 80 años) $1^{\circ}$ Edición - Perú: Fondo Editorial (Banco C.R. Perú).

Leddy, S. y Mae Pepper, J. (1989). Bases conceptuales de la enfermería profesional. New York - Estados Unidos: J. B. Lippincott

Marriner, T. Ann. (1999). Modelos y teorías de enfermería. 4. ${ }^{a}$ Edición. Edición en Español. Madrid - España: Elsevier.
Orem, Dorotea. (1999). Modelos y teorías de enfermería. Barcelona: Harcourt Brace.

Orem, D. E. (1994). Conceptos de enfermería en la práctica, México: Ediciones Científicas y Técnicas, S.A.

OMS - Organización Mundial de la Salud (2001). Régimen alimentario, actividad física y salud, 109 ${ }^{\mathrm{a}}$ reunión del Consejo Ejecutivo.

OMS - Organización Mundial de la Salud (2011). Enfermedades no transmisibles. Recuperado el 23 de junio del 2013 de: http://www.who.int/entity/fctc/es/ index.html.

Quintero, M. (1997). Autocuidado: Una estrategia eficaz en la atención del anciano, Lima: Trabajo presentado en I Congreso Internacional de Geriatría y Gerontología. Setiembre (mimeografiado).

Romero, C., Thelma, O. (2005). Conocimientos y prácticas sobre estilos de vida saludables en la enfermeras y auxiliares de enfermería del Servicio de Medicina de Infantes Hospital Roosevelt. (Tesis de Licenciatura). Universidad de San Carlos de 74 Guatemala.

Tobón, O. (2004). Fundamentos teóricos y metodológicos para el trabajo comunitario, $1 .^{\text {a }}$ edición, Universidad DeCaldas.ofetoc@telesat.com.co

Tobón, O. (2012). El autocuidado, una habilidad para vivir. (Tesis especialidad). Universidad de Caldas. Recuperado de: http://es.scribd.com/doc/51187879/ Autocuidado-Ofelia-Tobon-Correa

Uribe, J. (2003). El autocuidado y su papel en la promoción de la salud. (Tesis Maestría). Universidad de Antioquia. Colombia. Recuperado el 15 de mayo del 2013 de: http//caribe.udea.edu.co/rieenf/sep99/autocuidado.htm. http://tone.udea.edu.co./revista/sep99/autocuidado. htm.

Weinstein, N.D. (1988). The Precaution Process. Health Psychology, 4.355-386.

Recibido:30/06/2014 Aceptado: 01/10/2014 